\title{
Experimental technique for antiproton-nucleus annihilation cross section measurements at low energy
}

\author{
H. Aghai-Khozani ${ }^{1}$, M. Corradini ${ }^{2,3}$, R. Hayano ${ }^{4}$, M. Hori ${ }^{1,4}$, M. Leali ${ }^{2,3}$, E. Lodi-Rizzini ${ }^{2}$, \\ V. Mascagna ${ }^{2,3, a}$, Y. Murakami ${ }^{4}$, M. Prest ${ }^{5,6}$, L. Solazzi ${ }^{7,3}$, E. Vallazza ${ }^{8}$, L. Venturelli ${ }^{2,3}$, and \\ H. Yamada ${ }^{4}$ \\ ${ }^{1}$ Max-Planck-Institut für Quantenoptik, D-85748 Garching, Germany \\ ${ }^{2}$ Dipartimento di Ingegneria dell'Informazione, Università degli Studi di Brescia, I-25123 Brescia, Italy \\ ${ }^{3}$ Istituto Nazionale di Fisica Nucleare - Pavia, I-27100 Pavia, Italy \\ ${ }^{4}$ Department of Physics, University of Tokyo, Bunkyo-ku, Tokyo 113-0033, Japan \\ ${ }^{5}$ Dipartimento di Scienza e Alta Tecnologia, Università degli Studi dell'Insubria, I-22100 Como, Italy \\ ${ }^{6}$ Istituto Nazionale di Fisica Nucleare - Milano Bicocca, I-20126 Milano, Italy \\ ${ }^{7}$ Dipartimento di Ingegneria Meccanica e Industriale, Università degli Studi di Brescia, I-25123 Brescia, Italy \\ ${ }^{8}$ Istituto Nazionale di Fisica Nucleare - Trieste, I-34127 Trieste, Italy
}

\begin{abstract}
The interaction of very low energy antiprotons ( $\bar{p} \mathrm{~s})$ and antineutrons $(\bar{n} \mathrm{~s})$ with nuclei is interesting for its influence on both fundamental cosmology and nuclear physics. Measuring the annihilation cross section of antimatter on matter can help in solving the universe matter-antimatter puzzle and could give relevant hints in the definition of strong interaction model parameters as well.

The ASACUSA collaboration recently measured the antiproton-carbon annihilation cross section at $5.3 \mathrm{MeV}$ of kinetic energy of the incoming antiproton. The experimental apparatus consisted in a vacuum chamber containing thin foils $(\sim 0.7-1 \mu \mathrm{m})$ of carbon crossed by a bunched beam of antiprotons from the CERN Antiproton Decelerator (AD). The fraction of antiprotons annihilating on the target nucleons gives origin to charged pions which can be detected and counted by segmented scintillators placed outside the chamber. This work describes the experimental details of the apparatus and the technique to perform the cross section measurements.
\end{abstract}

\section{Introduction}

Nowdays the only source of antiprotons in the $\sim \mathrm{MeV}$ energy range is the CERN Antiproton Decelerator (AD) [1].

The CERN Proton Synchrotron protons are extracted towards the AD target where the emerging antiprotons are selected, collected and injected into the storage ring at $3.5 \mathrm{GeV}$. Then a deceleration down to $5.3 \mathrm{MeV}$ is performed and eventually the $\bar{p}$ s are delivered to the experimental areas in a bunch every $\sim 2$ minutes. A bunch lasts $200-400 \mathrm{~ns}$ and contains around $3 \times 10^{7}$ antiprotons.

Since the first circulation of antiprotons in AD, the ASACUSA Collaboration (Atomic Spectroscopy

\footnotetext{
${ }^{a}$ Now at Dipartimento di Scienza e Alta Tecnologia, Università degli Studi dell'Insubria, I-22100 Como, Italy and Istituto Nazionale di Fisica Nucleare - Milano Bicocca, I-20126 Milano, Italy
} 


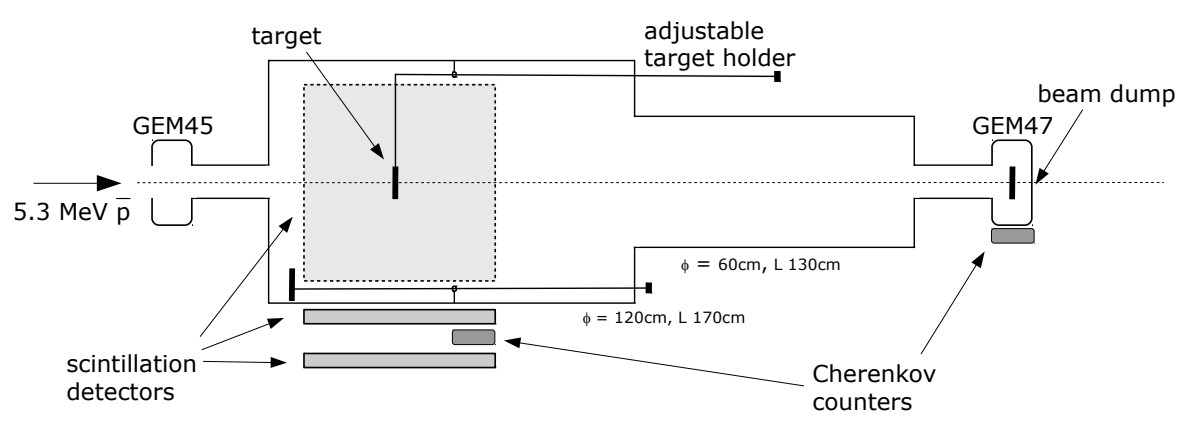

Figure 1. Experimental layout. A vacuum chamber contains the target held by a movable arm. Several scintillation detectors surround the chamber, and the apparatus is put within two movable beam profile monitors based on Gas Electron Multipliers (GEM45 and GEM47). The GEM47 is left on the beam axis position in order to stop the antiproton bunch.

And Collisions Using Slow Anti-protons) has performed experiments in different physics subjects: laser spectroscopy of antiprotonic helium atoms [3-5], microwave spectroscopy of antihydrogen ground-state hyper-fine structure [7-9], and nuclear collision cross section measurements of $\bar{p}$ at low energies ( 0.1-5 MeV) [10-15].

The existing experimental data of the annihilation cross section of antinucleons on nuclei show more than one discrepancy in the low energy region. For instance, if the same optical models which well describe the behavior of antineutrons and antiprotons at higher energies is used, the low energy extrapolated values are smaller than the measured ones; another strangeness is that the data for the antineutron seem to be well fitted by a function with a $1 / p^{2}$ dependence [6] which is common for low energy antiprotons, or more generally for charged particles (where a focusing effect due to the Coulomb attraction by the target nucleus is expected).

In order to provide useful data to solve these puzzles, the ASACUSA Collaboration has performed the measurement of the antiproton annihilation on carbon nucleus at $5.3 \mathrm{MeV}$, and this paper describes the experimental apparatus and technique used to achieve this goal.

\section{The apparatus}

A scheme of the experimental apparatus is shown in Fig. 1. Two cylindrical chambers (diameters of $120 \mathrm{~cm}$ and $60 \mathrm{~cm}$, lengths of $170 \mathrm{~cm}$ and $130 \mathrm{~cm}$, respectively) are connected toghether and put between two retractable Gas Electron Multipliers beam monitors (GEM45 and GEM47) used during the beam tuning to align the antiproton beam axis to the apparatus. Moreover, the second one (GEM47) is left in the on-axis position to dump the beam at the end.

Two Cherenkov counters, one at the target level, another one at the end of the beamline, are used to monitor the time structure and the intensity of the beam (see Sec. 2.3).

\subsection{The target system}

The two cylinders are made of stainless steel and form a unique vacuum chamber $\sim 3 \mathrm{~m}$ long wich is emptied at $3-4 \times 10^{-7}$ mbar. Inside the chambers 4 movable arms are available, each one supporting a target holder which can be moved both in the radial direction (i.e. in and out of the beam axis) and 
in the longitudinal one (parallel to the beam axis). The target holders, which consist in rings with an internal diameter of $11 \mathrm{~cm}$ and an external one of $13 \mathrm{~cm}$, are made of carbon fiber to match the target material.

The carbon targets are Diamond-like Carbon (DLC) self-supporting foils manufactured by Micromatter $^{1}$. They have been produced with thicknesses of $700 \mathrm{~nm}$ and $1000 \mathrm{~nm}$, according to the manufacturer with a precision of $2-3 \%$ and a (relative) uniformity of the target surface smaller than $2 \%$.

Two out of four of the target holders are used for a target, a third one is kept without any target (for evaluating the background) and the last holder has a different diameter to evaluate the elastic scattering contribution (see Sec. 3).

\subsection{The scintillating detector}

The scintillators surrounding the target chamber consist in 9 planes, 7 with an area of $\sim 1 \mathrm{~m}^{2}$ and 2 with an area of $\sim 0.5 \mathrm{~m}^{2}$. A variable number of scintillating bars ranging from 30 to 62 bars forms each plane. The bars are provided by FNAL ${ }^{2}$ and have a length of $96 \mathrm{~cm}$ and a section of $1.5 \times 1.9 \mathrm{~cm}^{2}$ (see Fig. 2).

They scintillator material is Polystyrene Dow Styron $663 \mathrm{~W}+1 \%$ PPO $+0.03 \%$ POPOP and each
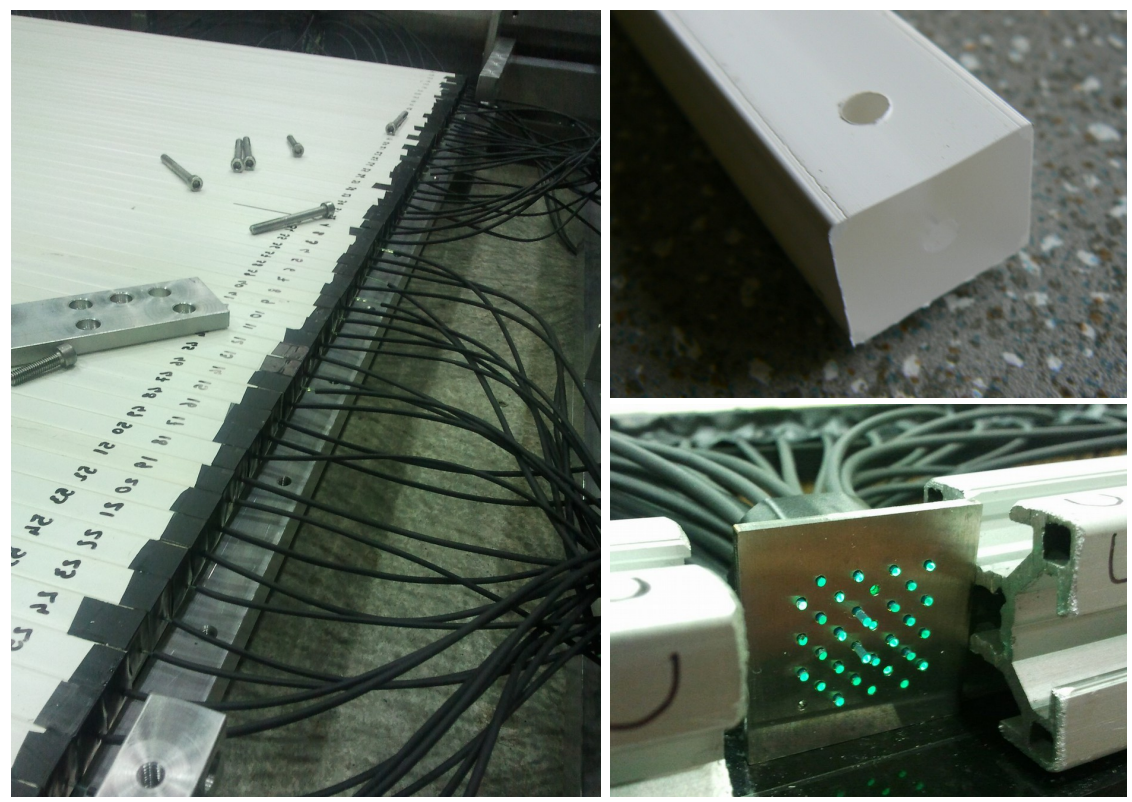

Figure 2. Right top: detail of the edge of one scintillating bar used in the detector: a hole along the bar axis is grooved to host the WLS fiber, while the one visible in the upper part is filled with glue once the fiber has been inserted. Right bottom: the $3 \times 3 \mathrm{~cm}^{2}$ aluminum plate used to match the fibers with the multianode PMT window. Left: a scintillator plane with 62 bars before closing the detector; the darkened WLS fibers are visibile.

bar has white $\mathrm{TiO}_{2}$ coating. Along the bar axis, a hole of $2.5 \mathrm{~mm}$ diameter is used to host a Y-11 type WLS fiber by Kuraray ${ }^{3}$ glued with E30 epoxy. The fiber diameter $(1 \mathrm{~mm})$ and the glue choice, are the

\footnotetext{
${ }^{1}$ http://www.micromatter.com/dlc.php

${ }^{2}$ http://www.fnal.gov

${ }^{3}$ http://www.kuraray.co.jp/en/
} 
results of dedicated tests [?].

The light collected by the WLS fibers is readout by multianode Hamamatsu 64 channel H7546-B photomultipliers (PMT in the following), which are installed into the scintillating bars frame matching the aluminum plates shown in Fig. 2. Despite the fact that every PMT allows the readout of 64 channels, actually only half of their channels have been coupled to a fiber (30 or 31 , depending on the detector) in a checkboard scheme to minimize the cross-talk between contiguous channels.

The analog signals coming from the PMTs are readout by custom front-end boards (FEB) based on the MAROC3 ASIC from LAL ${ }^{4}$. A single FEB is connected to one PMT with a dedicated socket and hosts 2 FPGAs (Altera Cyclone II) and a 12 bit ADC. The scheme of the MAROC3 chip and a picture of the board are shown in Fig. 3.

The MAROC3 processes in parallel the analog signals from the PMTs using pre-amplifiers with an
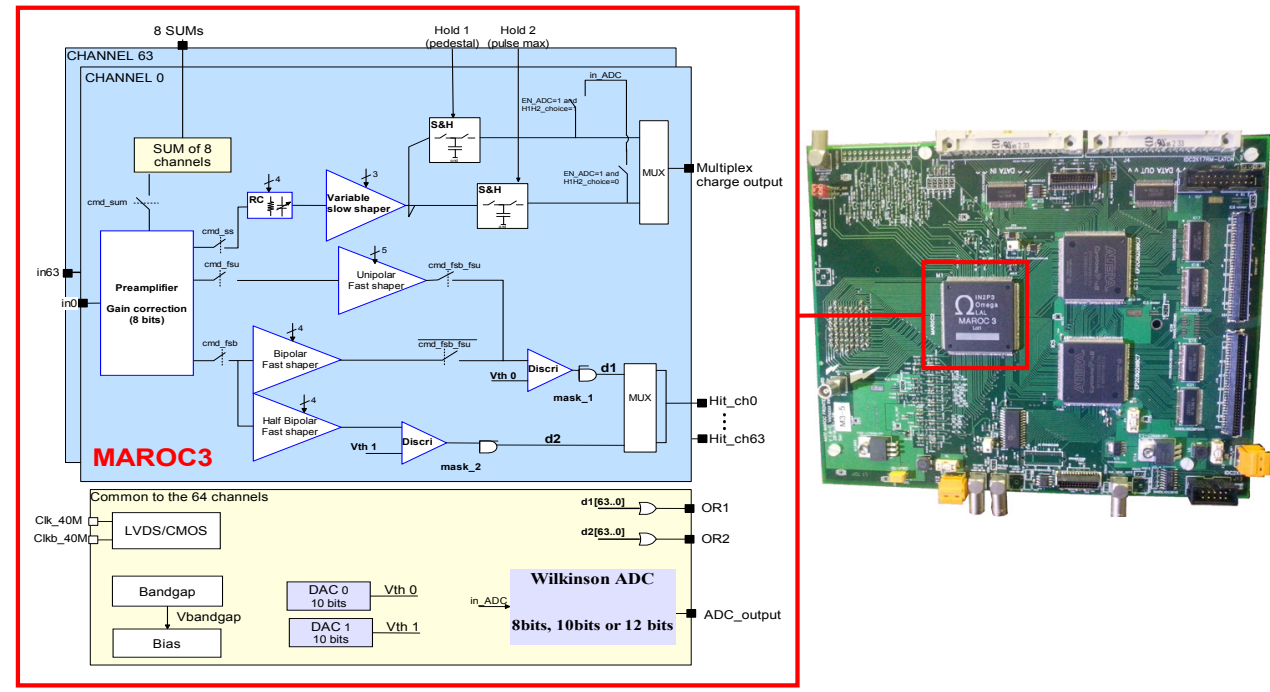

Figure 3. Right: a photo of one of the front-end boards which have been designed for the detector. Left: scheme of the MAROC3 chip (from the datasheet).

adjustable gain ( 8 bit). Then the chain is split in two parts: the first one, preserving the information on the analog pulse amplitude, consists of a slow shaper and a sample\&hold circuit; the second one is composed by a fast shaper and a discriminator. The analog outputs are multiplexed with a clock of $5 \mathrm{MHz}$ and digitized by the ADC on the board. The digital output, following the electronic scheme already used in the previous cross section measurements by the ASACUSA collaboration [16-19], is sampled by means of a $300 \mathrm{MHz}$ clock of the FPGAs to obtain the time information of each hit.

The FEB can be operated using either the analog ouputs or the digital one, respectively the "analog mode" and the "digital mode". In the present experiment the analog mode is used only for testing purposes, while the actual acquisition scheme makes use of the digital one following the scheme shown in Fig. 4. The FEB digital signals are handled by a set of VME buffer based custom electronics boards controlled by the data acquisition program (DAQ) which is ultimately driven by the AD machine trigger. Once a trigger is given to the DAQ, the shaped and discriminated PMT signals are sampled by a $300 \mathrm{MHz}$ clock (both on the rising and falling edge to double the time resolution) resulting in a $0 / 1$

\footnotetext{
${ }^{4}$ Laboratoire de l'Accélérateur Linéaire, Orsay. http://www.lal.in2p3.fr/
} 


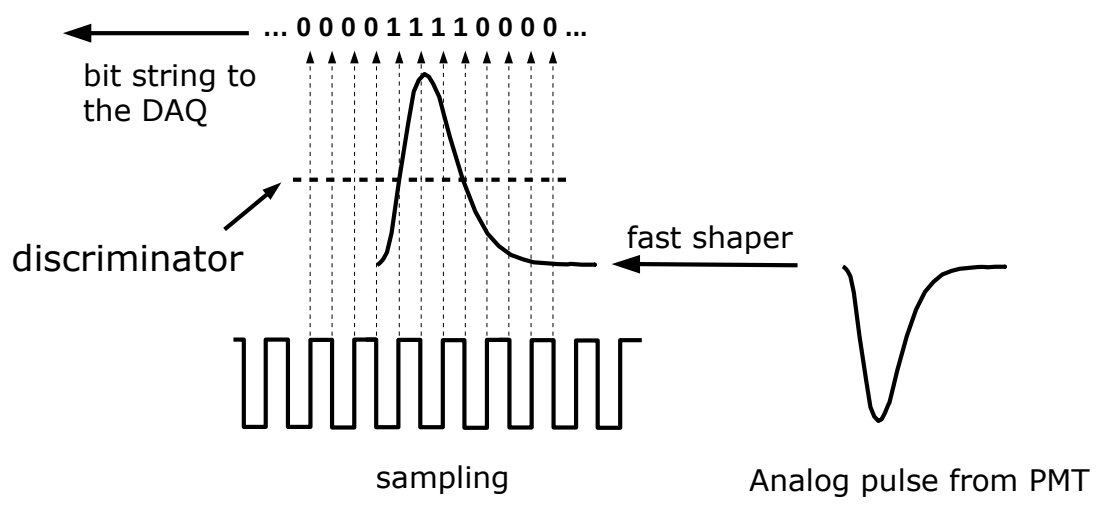

Figure 4. Details of the digital acquisition scheme. The output consists in a 512 bit string for each channel representing its time-over-threshold status.

when the signals are respectively below/above the discriminator threshold. The so formed bit string contains the information needed for the analysis, that is the instant the hit occured (starting point of the bit string) and its length (the time the signal is over threshold which is assumed to be proportional to the pulse height).

\subsection{The beam monitor detectors}

Two Cherenkov detectors have been used in two different positions (Fig. 1): the first one at the target level is used to study the time structure of the bunch (the destructive measurement is taken putting the target holder frame on the beam axis in order to completely stop the beam); in order to have a reliable monitor of the $\bar{p}$ beam intensity on a shot by shot basis, a second detector is put at the end of the beamline. The two Cherenkov detectors are made of lead fluoride crystals with a refractive index of $n=1.89$. The counters contain 5 crystals each one with a size of $30 \mathrm{~mm} \times 30 \mathrm{~mm} \times 160 \mathrm{~mm}$. Avalanche photodiodes by Hamamatsu (S8664-1010) with a $\sim 5 \mu$ m depletion layer were used to reduce background events caused by direct hits of charged particles at the depletion layers.

\section{Measurement technique}

The measurement technique is sketched in Fig. 5. Each antiproton bunch travels along the beamline crossing the thin carbon target where some antiprotons undergo in-flight annihilation. Despite the fact that this is the physical quantity to be measured, this number is expected to be a tiny fraction of the incoming $\bar{p}$ s, $10^{-6}$ or $10^{-5}$ depending on the used model. A fraction of antiprotons can also be elastically scattered by the target nuclei towards the lateral walls and eventually annihilate.

In both cases, the emerging annihilation charged products (mainly pions) are detected by the scintillation detectors placed close to the vacuum chamber containing the target, but the detection does not occur at the very same time since the pions from the annihilation of the scattered antiprotons are expected later in time (because of the time-of-flight of the antiprotons from the target to the lateral walls). Given the antiproton speed $(3 \mathrm{~cm} / \mathrm{ns}$ at $5.3 \mathrm{MeV})$ and the chamber diameter $(60 \mathrm{~cm}$, see 


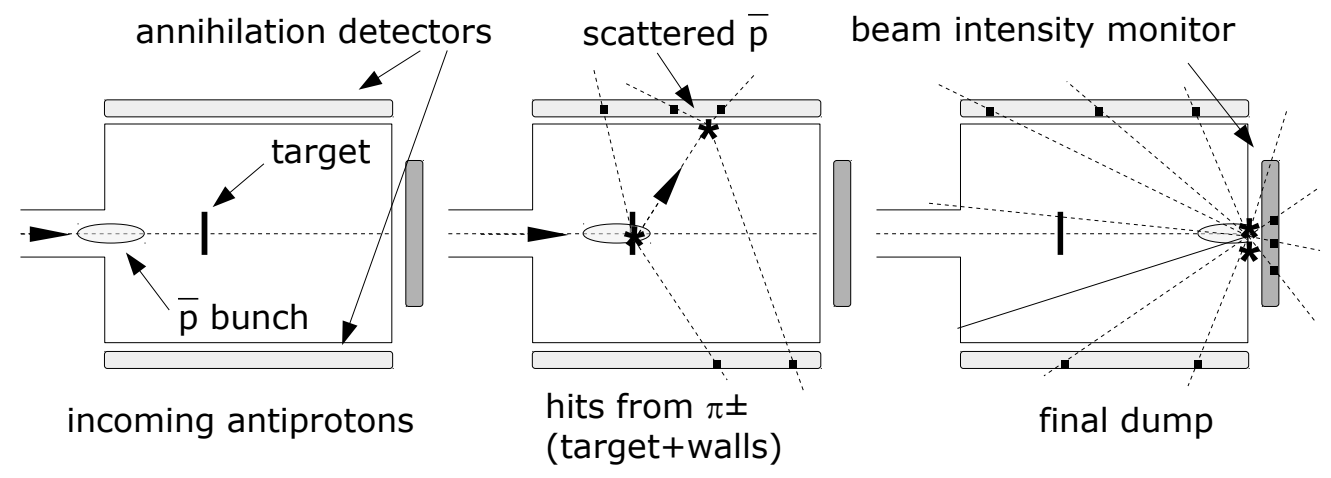

Figure 5. Scheme of the measurement technique (not to scale): the different moments of the experiment are shown as time increases from the left to the right: (left) the antiproton bunch $(50 \mathrm{~ns}$ long) is sent into the vacuum chamber, (middle) it crosses the carbon target and the first charged particles arising from the annihilation in the target and in the lateral walls are detected, (right) eventually the bunch arrives at the chamber end. See the text for a more detailed description.

Sec. 2), the time delay is at least $20 \mathrm{~ns}$, and this is a crucial parameter for the data analysis.

However, the vast majority of the $\bar{p}$ s cross at all with the target and travel to the end wall undisturbed where a measurement of the bunch intensity is performed by the Cherenkov detector described in Sec. 2 and, at the same time, the scintillating bars counters saturate.

To perform the measurement, a fiducial time window corresponding to the first $20 \mathrm{~ns}$ of the annihilations in the target region is selected and the hits detected in this window are counted.

In order to compute the annihilation cross section, the number of incoming antiprotons must be taken into account. A normalization on a shot by shot basis is done by means of the already mentioned Cherenkov detector (Sec. 2), while the absolute number of incoming $\bar{p}$ s is determined using a second ring-shaped frame (similar to the target support) as shown in Fig. 6. Being both the Coulomb scat-

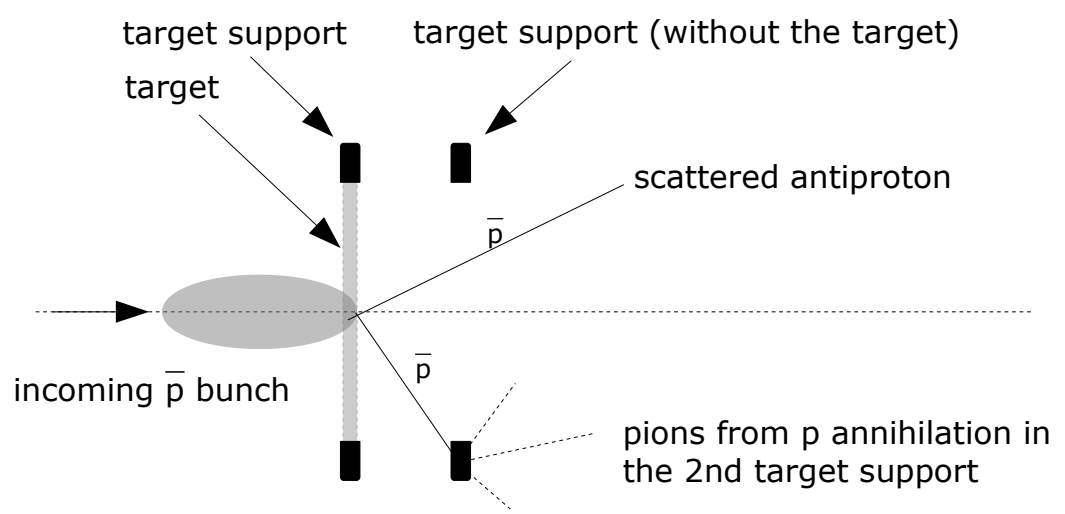

Figure 6. Layou of the "second frame" technique to measure the incoming antiproton number. A known fraction of scattered $\bar{p}$ is intercepted by the ring and the annihilations are detected (with the ones in the target) in dedicated runs. 
tering cross-section and the fraction of solid angle seen by the center of the target well known, it is possible to calibrate the beam monitor detector (see a more detailed desctiption in $[12,20]$ ).

The final used formula for the annihilation cross section of antiprotons on carbon is:

$$
\sigma_{\text {ann }}=\frac{N_{\text {target }}}{N_{\text {ring }}} \sigma_{\text {scatt }}
$$

where $N_{\text {target }}$ is the number of events detected when the target is placed on the beam axis, $N_{\text {ring }}$ the ones when the "second ring" is added and $\sigma_{\text {scatt }}$ the Coulomb multiple scattering cross section integrated over the solid angle intercepted by the "second ring".

\section{Conclusion}

The apparatus and technique to perform the measurement of the annihilation cross section of $5.3 \mathrm{MeV}$ antiprotons on a carbon nucleus have been described. The experiment has been performed at the CERN Antiproton Decelerator in November 2015, the preliminary results have been published [21]; for a full analysis discussione see [22, 23].

\section{Acknowledgements}

This work was supported by Istituto Nazionale di Fisica Nucleare (INFN), Università di Brescia, MEXT Japan (grant no. 20002003), the European Research Council (ERC-StG) and the Max-PlanckGesellschaft. Special thanks to the staff of the CERN Antiproton Decelerator and Proton Synchrotron.

\section{References}

[1] The Antiproton Decelerator, CERN 2012 http://home.cern/about/accelerators/antiproton-decelerator

[2] ASACUSA Collaboration webpage http://www.cern.ch/ASACUSA

[3] M. Hori, et al., Nature 475484 (2011)

[4] R. S. Hayano et al., Rep. Prog. Phys. 701995 (2007)

[5] M. Hori, et al., Science 3546312 610-614 (2016)

[6] M. Astrua et al., Nucl. Phys. A 697 209-224 (2002)

[7] Y. Enomoto et al., Phys. Rev. Lett. 105243401 (2010)

[8] N. Kuroda et al., Nature Comm. 53089 (2014)

[9] N. Kuroda et al., Hyp. Int. 235 13-20 (2015)

[10] A. Bianconi et al., Phys. Lett. B 704461 (2011)

[11] M. Corradini et al., Nucl. Instr. Methods A 71112 (2013)

[12] H. Aghai-Kohzani et al., 12710 1-6 (2012)

[13] M. Corradini et al., Hyp. Int. 233 53-58 (2015)

[14] H. Aghai-Kohzani et al., EPJ Web Conf. 6609001 (2014)

[15] H. Aghai-Kohzani et al., Hyp. Int. 234 85-92 (2015)

[16] V. Mascagna et al., Nucl. Phys. B 172299 (2007)

[17] A. Mozzanica et al., Il Nuovo Cimento 122 B 6 (2007)

[18] M. Corradini et al., Hyp. Int. 194305 (2009) 
[19] M. Corradini et al., Hyp. Int. 21331 (2012)

[20] K. Todoroki et al., Nucl. Instr. Methods A 835 110-118 (2016)

[21] H. Aghai-Kohzani et al., EPJ Web Conf. 130, 07014 (2016)

[22] H. Aghai-Khozani et al., sumbitted to Nucl. Phys. A, (2017)

[23] H. Aghai-Khozani et al., these proceedings, (2017) 